\title{
Resolving Spacetime Singularities within Asymptotic Safety
}

\author{
Lando Bosma, ${ }^{*}$ Benjamin Knorr, ${ }^{\dagger}$ and Frank Saueressig $\odot^{\ddagger}$ \\ Institute for Mathematics, Astrophysics and Particle Physics (IMAPP), Radboud University Nijmegen, \\ Heyendaalseweg 135, 6525 AJ Nijmegen, Netherlands
}

(Received 19 April 2019; published 5 September 2019)

\begin{abstract}
A key incentive of quantum gravity is the removal of spacetime singularities plaguing the classical theory. We compute the nonperturbative momentum dependence of a specific structure function within the gravitational asymptotic safety program which encodes the quantum corrections to the graviton propagator for momenta above the Planck scale. The resulting quantum-corrected Newtonian potential approaches a constant negative value as the distance between the two point masses goes to zero, thereby removing the classical singularity. The generic nature of the underlying mechanism suggests that it will remain operative in the context of black hole and cosmic singularities.
\end{abstract}

DOI: 10.1103/PhysRevLett.123.101301

General relativity provides a well-established theory for gravity from submillimeter up to cosmic scales [1]. It has been extremely successful in predicting phenomena like the bending of light by a gravitational field, the gravitational redshift of photons, or the existence of gravitational waves. Another striking feature of general relativity is that its solutions rather generically contain specific points where the curvature of spacetime diverges, so-called singularities [2]. Well-known examples are the curvature singularities of classical black holes. This feature is often paraphrased as "general relativity predicting its own breakdown" [3] and provides one of the central motivations for the search of a more complete theory of gravity, commonly referred to as "quantum gravity." Conversely, any candidate for such a theory should explain the fate of these spacetime singularities. In loop quantum gravity, black hole singularities may be removed by quantum geometry effects [4-6]; also see [7] for a recent review. Similarly, the fuzzball proposal $[8,9]$ provides a mechanism for obtaining regular black holes in the framework of string theory. For the gravitational asymptotic safety program [10-14], the method of renormalization group improvement suggests that black hole singularities may be removed by quantum effects $[15,16]$; also see $[17,18]$ for the current status and further references. The present work takes a key step towards understanding the fate of spacetime singularities within asymptotic safety, based on a first-principles computation. Our main finding is displayed in Fig. 3, showing that the short-distance divergence in the spin-2 channel of

Published by the American Physical Society under the terms of the Creative Commons Attribution 4.0 International license. Further distribution of this work must maintain attribution to the author(s) and the published article's title, journal citation, and DOI. Funded by SCOAP ${ }^{3}$.
Newton's gravitational potential is resolved by quantum gravity effects.

In order to exhibit this effect, we follow the path taken in the effective field theory treatment of quantum gravity $[19,20]$ and construct the gravitational potential $V(r)$ arising from the one-graviton exchange between two scalar fields with masses $m_{1}$ and $m_{2}$ minimally coupled to gravity. Taking the static limit where the two scalars have infinite mass, one has $[20,21]$

$$
V(r)=-\frac{1}{2 m_{1}} \frac{1}{2 m_{2}} \int \frac{d^{3} \mathbf{q}}{(2 \pi)^{3}} e^{i \mathbf{q} \cdot \mathbf{r}} \mathcal{M}
$$

Denoting Newton's coupling by $G$, the scattering amplitude associated with the Feynman diagram (Fig. 1) evaluated in general relativity is $\mathcal{M}=16 \pi G m_{1}^{2} m_{2}^{2} /|\mathbf{q}|^{2}$, evaluated for the nonrelativistic limit of the propagator $q^{\alpha}=(0, \mathbf{q})$. Evaluating the Fourier integral, one recovers the classical Newtonian gravitational potential $V_{c}(r)=-G m_{1} m_{2} / r$.

In the following, we will focus on the contribution of the transverse-traceless (spin-2) mode to $\mathcal{M}$. [The complete analysis should also include the quantum corrections to the gravitational propagator for the spin- 0 mode. This requires adding the structure function $R f_{k}(\Delta) R$ to the ansatz for Eq. (4) encoding the nontrivial momentum dependence of

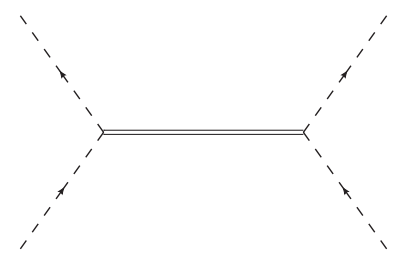

FIG. 1. Tree-level amplitude describing the interaction of two scalars of mass $m_{1}$ and $m_{2}$ (dashed lines) due to the exchange of a graviton (double line). 
the scalar propagator. This analysis is beyond the scope of this work and will be presented elsewhere [22]. We expect that this will give rise to similar corrections as the ones entering (5).] Performing the tensor contractions and taking the static limit, one finds

$$
\mathcal{M}^{\mathrm{TT}}=\frac{64 \pi G}{3} m_{1}^{2} m_{2}^{2} \mathcal{G}^{\mathrm{TT}}\left(\mathbf{q}^{2}\right),
$$

where $\mathcal{G}^{\mathrm{TT}}$ is the scalar part of the spin-2 propagator carrying the momentum dependence and is obtained from the full propagator by a contraction with the transversetraceless projector. For the Einstein-Hilbert action, $\mathcal{G}^{\mathrm{TT}}=$ $1 / q^{2}$ so that the resulting potential $V_{c}^{\mathrm{TT}} \propto 1 / r$ diverges as $r \rightarrow 0$. In this sense, the nonrelativistic limit already includes many of the essential features related to the curvature singularity encountered in black hole physics.

Treating gravity as an effective field theory allows us to compute the leading (long-distance) quantum corrections to $V_{c}(r)$ using perturbation theory $[19,20]$. These corrections do not resolve the divergence in $V_{c}(r)$, though. Equation (2) then suggests to compute the nonperturbative propagator $\mathcal{G}^{\mathrm{TT}}\left(\mathbf{q}^{2}\right)$ and to investigate the resulting shortdistance behavior of the quantum-corrected potential $V_{q}^{\mathrm{TT}}(r)$. In this work, we perform such a computation within the gravitational asymptotic safety program.

Structure functions for gravity.-A canonical tool for computing properties of a quantum field theory beyond the realm of perturbation theory is the Wetterich equation $[23,24]$

$$
k \partial_{k} \Gamma_{k}=\frac{1}{2} \operatorname{Tr}\left[\left(\Gamma_{k}^{(2)}+\mathcal{R}_{k}\right)^{-1} k \partial_{k} \mathcal{R}_{k}\right],
$$

governing the change of the effective average action $\Gamma_{k}$ when quantum fluctuations around the momentum scale $k$ are integrated out. Here, $\Gamma_{k}^{(2)}$ denotes the second functional derivative of $\Gamma_{k}$ with respect to the fluctuation field, $\mathcal{R}_{k}$ is a suitable regulator function which provides a mass term for fluctuations with momenta $p^{2}<k^{2}$ and vanishes for $p^{2} \gg k^{2}$, and the trace contains a sum over fluctuation fields as well as an integral over loop momentum. By now, the Wetterich equation has proven its merits in statistical physics [25], nonequilibrium physics [26], and gauge theories [27]. Starting from the pioneering work in Ref. [28], which introduced the functional renormalization group in the context of gravity, there is now solid evidence supporting the existence of a nontrivial renormalization group fixed point for four-dimensional gravity [11,29-50] and many gravity-matter systems potentially including the standard model of particle physics [13,51-62]. In particular, the full momentum dependence of the gravitational propagators starting from $\Gamma_{k}$ given by the Einstein-Hilbert action has been studied in Refs. [63,64]. Assuming that this fixed point controls the short-distance behavior of the gravitational interaction for lengths $\ell$ smaller than the Planck length $\ell_{\mathrm{Pl}} \approx$ $10^{-35} \mathrm{~m}$ would put gravity in the class of nonperturbatively renormalizable quantum field theories along the lines of Weinberg's asymptotic safety scenario [65].

A virtue of Wetterich's equation (3) is that one can extract nonperturbative information about a given quantum theory by making a suitable ansatz for $\Gamma_{k}$ and studying the flow of the effective average action on the corresponding subspace. A suitable ansatz capturing the momentum dependence of the gravitational propagator involves scaledependent structure functions acting on curvature tensors. In this work, we will focus on the nontrivial momentum dependence of the spin-2 propagator captured by

$$
\Gamma_{k}^{\mathrm{grav}}=\frac{1}{16 \pi G_{k}} \int d^{4} x \sqrt{g}\left[-R+2 \Lambda_{k}+C_{\mu \nu \rho \sigma} W_{k}(\Delta) C^{\mu \nu \rho \sigma}\right] .
$$

Here, $R, C_{\mu \nu \rho \sigma}$, and $\Delta \equiv-g^{\mu \nu} D_{\mu} D_{\nu}$ denote the Ricci scalar, Weyl tensor, and Laplacian constructed from the spacetime metric $g_{\mu \nu}$, respectively. Furthermore, the ansatz contains a scale-dependent Newton's coupling $G_{k}$ and cosmological constant $\Lambda_{k}$ as well as the scale-dependent structure function $W_{k}(\Delta)$. Expanding (4) in fluctuations around flat space and restricting the result to the transverse-traceless sector yields the graviton propagator

$$
\mathcal{G}^{\mathrm{TT}}\left(q^{2}\right)=\left[q^{2}+2\left(q^{2}\right)^{2} W_{k}\left(q^{2}\right)\right]^{-1} .
$$

Thus, the structure function $W_{k}\left(q^{2}\right)$ captures nontrivial corrections to the graviton propagator. The Einstein-Hilbert result is recovered by setting $W_{k}\left(q^{2}\right)=0$.

The scale dependence of $G_{k}, \Lambda_{k}$, and $W_{k}\left(q^{2}\right)$ can be obtained by supplementing the ansatz (4) by suitable gauge-fixing and ghost terms, substituting the resulting expression into Wetterich's equation (3), and projecting the trace on the subspace spanned by the ansatz. The calculation of the flow equations for Newton's coupling and the cosmological constant takes into account the full fluctuation spectrum. Owing to the formidable complexity of the computation, the flow of $W_{k}$ is evaluated in the conformally reduced setting $[66,67]$, where the right-hand side of the flow equation retains the fluctuations of the conformal mode only. In this case, the spacetime metric $g_{\mu \nu}$ is taken to be of the form $g_{\mu \nu}=[1+(1 / 4) h] \hat{g}_{\mu \nu}$, where $h$ is the fluctuation field and $\hat{g}_{\mu \nu}$ is a fixed but arbitrary reference metric. From analogous computations in the framework of $f(R)$ gravity [37,68-71], it is expected that the resulting qualitative behavior of the structure function matches the one obtained from including all metric fluctuations.

Our goal is to find a self-consistent flow equation retaining the full information on the functional form of $W_{k}(\Delta)$, i.e., without making approximations related to the 
momentum dependence. We achieve this goal by combining two computational techniques tailored to the two classes of curvature terms appearing in the trace evaluation. Terms containing less than two powers of a (potentially contracted) Riemann tensor are evaluated using Mellin transform techniques [72] together with the nonlocal heatkernel results [73]. Terms containing two powers of the Weyl tensor are evaluated using flat-space momentumspace techniques. [The technical details on how the exact momentum dependence is retained and the conceptual relation between the structure function $W_{k}(\Delta)$ and the momentum-dependent anomalous dimensions $\eta\left(p^{2}\right)$ studied within the vertex expansion of $\Gamma_{k}[50,63,64,74]$ are provided in Ref. [75].] The resulting flow equations are conveniently expressed in terms of the dimensionless, scale-dependent couplings

$$
g \equiv G_{k} k^{2}, \quad \lambda \equiv \Lambda_{k} k^{-2}, \quad w\left(q^{2}\right) \equiv k^{-2} W_{k}\left(\Delta / k^{2}\right) .
$$

Neglecting the contribution of the structure function, the flow in the Einstein-Hilbert sector is governed by $[28,76]$

$$
\begin{aligned}
& k \partial_{k} \lambda=\left(\eta_{N}-2\right) \lambda+\frac{g}{2 \pi}\left[10 \Phi_{2}^{1}(-2 \lambda)-8 \Phi_{2}^{1}(0)-5 \eta_{N} \tilde{\Phi}_{2}^{1}(-2 \lambda)\right], \\
& k \partial_{k} g=\left(2+\eta_{N}\right) g,
\end{aligned}
$$

with the anomalous dimension of Newton's coupling $\eta_{N} \equiv$ $k \partial_{k} \ln G_{k}$ being given by

$$
\eta_{N}=\frac{\frac{g}{3 \pi}\left[5 \Phi_{1}^{1}(-2 \lambda)-18 \Phi_{2}^{2}(-2 \lambda)-4 \Phi_{1}^{1}(0)-6 \Phi_{2}^{2}(0)\right]}{1+\frac{g}{6 \pi}\left[5 \tilde{\Phi}_{1}^{1}(-2 \lambda)-18 \tilde{\Phi}_{2}^{2}(-2 \lambda)\right]} .
$$

The threshold functions

$$
\begin{aligned}
& \Phi_{n}^{p}(\mu)=\frac{1}{\Gamma(n)} \int_{0}^{\infty} d z z^{n-1} \frac{R(z)-z R^{\prime}(z)}{[z+R(z)+\mu]^{p}}, \\
& \tilde{\Phi}_{n}^{p}(\mu)=\frac{1}{\Gamma(n)} \int_{0}^{\infty} d z z^{n-1} \frac{R(z)}{[z+R(z)+\mu]^{p}}
\end{aligned}
$$

contain the dimensionless profile function $R(z)$ related to $\mathcal{R}_{k}$ [28].

The flow of $w\left(q^{2}\right)$ is treated in the conformally reduced approximation. This leads to the linear integro-differential equation

$$
\begin{aligned}
k \partial_{k} w\left(q^{2}\right)= & \left(2+\eta_{N}\right) w\left(q^{2}\right)+2 q^{2} w^{\prime}\left(q^{2}\right)+\frac{g}{24 \pi} \int_{0}^{1 / 4} d u(1-4 u)^{3 / 2} \frac{\left(2-\eta_{N}\right) R\left(u q^{2}\right)-2 u q^{2} R^{\prime}\left(u q^{2}\right)}{u q^{2}+R\left(u q^{2}\right)-\frac{4}{3} \lambda} \\
& +\frac{16 g}{3 \pi^{2}} \int_{0}^{\infty} d p \int_{-1}^{1} d x p^{3} \sqrt{1-x^{2}} \frac{\left(2-\eta_{N}\right) R\left(p^{2}\right)-2 p^{2} R^{\prime}\left(p^{2}\right)}{\left[p^{2}+R\left(p^{2}\right)-\frac{4}{3} \lambda\right]^{2}}\left[\frac{1}{8}\left[w\left(p^{2}+2 p q x+q^{2}\right)-w\left(q^{2}\right)\right]\right. \\
& +\frac{2 q^{4}+4\left(q^{2}-p^{2}\right)(p q x)+p^{2} q^{2}\left(7-6 x^{2}\right)}{16\left(p^{2}+2 p q x\right)^{2}}\left[w\left(p^{2}+2 p q x+q^{2}\right)-w\left(q^{2}\right)\right] \\
& \left.+\frac{3 p^{4}-2 q^{4}+22 p^{2}(p q x)-5 p^{2} q^{2}\left(1-6 x^{2}\right)}{16\left(p^{2}+2 p q x\right)} w^{\prime}\left(q^{2}\right)\right] .
\end{aligned}
$$

Here, the primes denote derivatives with respect to the argument, and $q$ is the dimensionless external momentum. The inhomogeneous term appearing in the first line originates from the Einstein-Hilbert sector. Thus, the quantum fluctuations from classical gravity will induce a nontrivial structure function $w\left(q^{2}\right)$ unless $g=0$. While the denominators in the square brackets suggest that the equation could contain collinear divergences, expanding the integrand at these points shows that this is not the case. All potential poles are canceled by zeros of the numerator.

It is straightforward to see that the system has a trivial fixed point $g_{*}=\lambda_{*}=w_{*}\left(q^{2}\right)=0$. Our primary interest is in nontrivial fixed point solutions $\left(g_{*}, \lambda_{*}, w_{*}\left(q^{2}\right)\right)$ of Eqs. (7) and (10) where, by definition, the couplings become independent of $k$. Equation (7) entails that at such a fixed point $\eta_{N}=-2$. Substituting this value into Eq. (10), one finds that the resulting fixed point equation is invariant under a constant shift of $w$. Thus, the equation contains one free parameter, which will be denoted by $w_{\infty}$. This freedom constitutes an artifact of the conformally reduced approximation and does not persist once fluctuations of transversetraceless modes are included. In order to obtain the global form of the structure function $w_{*}\left(q^{2}\right)$, we first perform an asymptotic expansion of Eq. (10) at infinite momentum. This establishes the leading-order behavior

$$
w_{*}\left(q^{2}\right) \underset{q \rightarrow \infty}{\sim} w_{\infty}+\frac{\rho}{q^{2}}+\cdots .
$$

The parameter $w_{\infty}$ fixes the value of $w_{*}\left(q^{2}\right)$ at asymptotically large momenta, and $\rho$ is a regulator-dependent positive number. 
The system of fixed point equations can then be further analyzed numerically. For this purpose, we resort to the regulator $R(z)=e^{-\alpha z}$. Importantly, this regulator is smooth and leads to a rapid convergence when the threshold integrals are evaluated numerically. All numerical values and illustrations are obtained with $\alpha=1$, and we checked that all results are robust with respect to changing $\alpha$.

Since the Einstein-Hilbert sector is independent of $w$, its fixed point structure can be analyzed before solving Eq. (10). It permits a non-Gaussian fixed point at $g_{*}=0.374, \lambda_{*}=0.285$. This fixed point acts as an ultraviolet attractor for the renormalization group flow in the $g-\lambda$ plane.

The global solution for $w_{*}\left(q^{2}\right)$ is then obtained through pseudospectral methods $[77,78]$ using rational Chebyshev functions as a basis set $[79,80]$. This leads to the solution shown in Fig. 2. This result has a number of remarkable properties. First, the solution is globally well defined and unique up to the constant $w_{\infty}$. The structure function interpolates between a constant for low momenta and the asymptotic behavior (11) for large momenta. The crossover occurs for the dimensionless momentum $q^{2} \approx 1$. Second, the solution is positive definite for all values $w_{\infty}>0$. This entails that the flat-space propagator (5) has a single first-order pole at $q^{2}=0$. In particular, there are no additional poles for $q^{2}>0$. While it would be interesting to extend the analysis of the pole structure to the complex plane, this is beyond the scope of this Letter. Third, the propagator grows polynomially only for asymptotically large momenta, indicating that the resulting theory is actually local. For the asymptotic parameter, we find $\rho \approx 0.0149$.

Remarkably, the numerical solution can be parametrized with very high precision by

$$
w_{*}^{\mathrm{fit}}\left(q^{2}\right) \approx \frac{\rho}{\frac{\rho}{\kappa}+q^{2}}+w_{\infty}, \quad \kappa \approx 0.00817 .
$$

We expect that this analytic approximation will be very useful when analyzing properties of the quantum theory in the future.

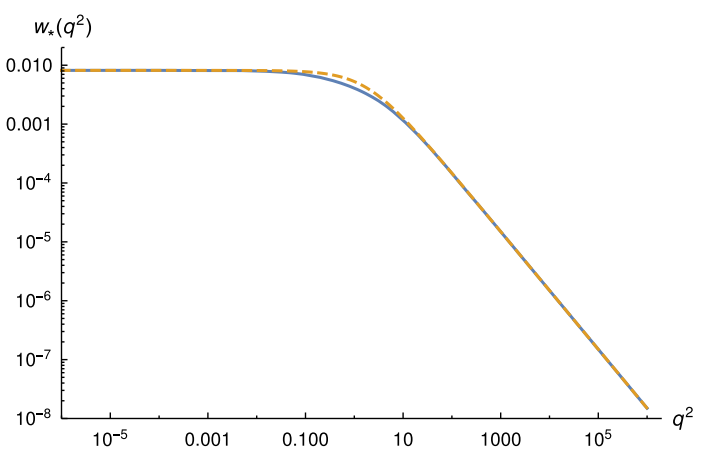

FIG. 2. Fixed function $w_{*}\left(q^{2}\right)$ for $w_{\infty}=0$ (orange, solid line). The parameterization (12) is superimposed as a dashed line.
The stability analysis should not be extended to structure functions related to propagators. Conceptually, such structure functions ought to be considered as a part of a momentum-dependent wave function renormalization. The critical properties of these structure functions are thus related to a momentum-dependent generalization of the anomalous dimension rather than to the critical exponents; see [63]. (We thank J. M. Pawlowski for discussion on this point.)

Quantum-corrected Newtonian potential.-As a first application of our computation, we calculate the quantum-corrected Newtonian potential $V_{q}^{\mathrm{TT}}(r)$ by evaluating Eq. (1) for the quantum-corrected flat-space propagator. This requires reintroducing a scale in $w\left(q^{2}\right)$. The analysis $[47,76,81,82]$ then indicates that $k^{2}$ should be identified with the observed value for Newton's coupling $G^{-1}$, which implies that the transition displayed in Fig. 2 occurs at the Planck scale. While this procedure may miss nonanalytic contributions to $w\left(q^{2}\right)$ arising from integrating the renormalization group flow to the infrared, it is clear that the fixed point will control the short-distance behavior, so that we can make reliable statements about this quantum-gravitydominated regime.

The central result is illustrated in Fig. 3. For distances larger than the Planck scale, the classical and quantum Newtonian potentials essentially coincide. For $w_{\infty}>0$, the quantum corrections to the propagator remove the shortdistance singularity in the classical Newton potential, however, such that $\lim _{r \rightarrow 0} V_{q}^{\mathrm{TT}}(r)$ is actually finite. This entails that the gravitational binding energy is bounded by $E_{\text {binding }}^{\mathrm{TT}}=-\left.V_{q}^{\mathrm{TT}}(r)\right|_{r=0}$. The parameterization (12) then allows us to compute this value

$$
E_{\text {binding }}^{\mathrm{TT}}=\frac{2}{3 w_{\infty}} \frac{1+\frac{\rho}{\sqrt{x_{+} x_{-}}}}{\sqrt{x_{+}}+\sqrt{x_{-}}} G m_{1} m_{2}
$$

where

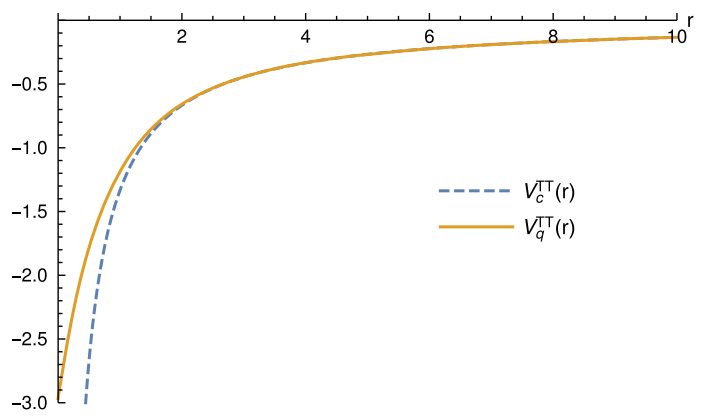

FIG. 3. Comparison of $V_{c}^{\mathrm{TT}}(r)$ (blue, dashed line) and $V_{q}^{\mathrm{TT}}(r)$ obtained from the quantum-corrected propagator with $w_{\infty}=0.1$ and $m_{1}=m_{2}=G=1$. For $w_{\infty}>0$, corrections to the highmomentum behavior resolve the divergence in the classical case, making the quantum-corrected potential $V_{q}(r)$ finite as $r \rightarrow 0$. 


$$
\begin{aligned}
x_{ \pm}= & \frac{1}{4 w_{\infty} \kappa}\left[\kappa+2\left(w_{\infty}+\kappa\right) \rho\right. \\
& \left. \pm \sqrt{\left.\left[\kappa+2\left(w_{\infty}+\kappa\right) \rho\right]^{2}-8 w_{\infty} \kappa \rho\right]}\right]
\end{aligned}
$$

The classical analysis [83] establishes that the resolution of the $r=0$ divergence is actually independent of the precise form of the structure function. Generically, any positive structure function will lead to complex mass poles which ensure that $E_{\text {binding }}^{\mathrm{TT}}$ is finite. Deriving the finiteness of $V_{c}^{\mathrm{TT}}$ from a first-principles computation is highly nontrivial and constitutes a major test of the underlying quantum theory. We expect that this will have drastic consequences for our understanding of spacetime singularities also in more general cases. In particular, it was argued in Ref. [84] that complex mass poles in the gravitational propagator could be associated with extended objects which could screen spacetime singularities from being probed by physical processes, thus leading to singularity avoidance in the context of black hole physics.

Conclusions.-This work constitutes a major step towards computing the quantum-corrected propagators in asymptotic safety. The nonperturbative short-distance corrections to the Newtonian potential shown in Fig. 3 outline the path for resolving the spacetime singularities plaguing classical gravity. This result differs from the perturbative treatment of gravity as an effective field theory [19,20], since the propagator underlying $V_{q}^{\mathrm{TT}}(r)$ is manifestly nonperturbative. In principle, the modifications in the Newtonian potential can be tested experimentally (see [85] for a related discussion), even though probing Newton's law on Planckian scales is far beyond current experimental possibilities.

Naturally, our findings bear a close connection to the ghost-free, nonlocal gravity program [86-89] and to noncommutative geometry [90-92], where structure functions of the type (4) play a key role. In nonlocal, ghost-free gravity, they constitute an input, defining the fundamental action, while the noncommutative geometry approach generates these terms through the nonlocal heat kernel. In both cases, the structure function exhibits an exponential falloff at momentum scales above the nonlocality scale. In Refs. [93,94], this has been paraphrased as "high-energy bosons do not propagate." The result of our first-principles computation differs qualitatively from these constructions, as the quantum-corrected propagator arising from (10) grows as $q^{4}$ for large momenta. This suggests that these approaches are in a different universality class than our (microscopically) manifestly local theory.

We thank F. Filthaut, S. Lippoldt, and C. Ripken for interesting discussions. This research is supported by the Netherlands Organisation for Scientific Research (NWO) within the Foundation for Fundamental Research on Matter (FOM) Grant No. 13VP12. *1.bosma@science.ru.nl

†b.knorr@science.ru.nl

f.saueressig@science.ru.nl

[1] C. M. Will, The confrontation between general relativity and experiment, Living Rev. Relativity 17, 4 (2014).

[2] S. W. Hawking and R. Penrose, The singularities of gravitational collapse and cosmology, Proc. R. Soc. A 314, 529 (1970).

[3] General Relativity-an Einstein Centenary Survey, edited by $\mathrm{S}$. W. Hawking and W. Israel (Cambridge University Press, Cambridge, England, 1979).

[4] A. Ashtekar and M. Bojowald, Black hole evaporation: A paradigm, Classical Quantum Gravity 22, 3349 (2005).

[5] A. Ashtekar and M. Bojowald, Quantum geometry and the Schwarzschild singularity, Classical Quantum Gravity 23, 391 (2006).

[6] L. Modesto, Black hole interior from loop quantum gravity, Adv. High Energy Phys. 2008, 459290 (2008).

[7] A. Barrau, K. Martineau, and F. Moulin, A status report on the phenomenology of black holes in loop quantum gravity: Evaporation, tunneling to white holes, dark matter and gravitational waves, Universe 4, 102 (2018).

[8] O. Lunin and S. D. Mathur, AdS/CFT duality and the black hole information paradox, Nucl. Phys. B623, 342 (2002).

[9] S. D. Mathur, The fuzzball proposal for black holes: An elementary review, Fortschr. Phys. 53, 793 (2005).

[10] M. Niedermaier and M. Reuter, The asymptotic safety scenario in quantum gravity, Living Rev. Relativity 9, 5 (2006).

[11] M. Reuter and F. Saueressig, Quantum Einstein gravity, New J. Phys. 14, 055022 (2012).

[12] R. Percacci, An Introduction to Covariant Quantum Gravity and Asymptotic Safety, 100 Years of General Relativity Vol. 3 (World Scientific, Singapore, 2017).

[13] A. Eichhorn, An asymptotically safe guide to quantum gravity and matter, Front. Astron. Space Sci. 5, 47 (2019).

[14] M. Reuter and F. Saueressig, Quantum Gravity and the Functional Renormalization Group (Cambridge University Press, Cambridge, England, 2019).

[15] A. Bonanno and M. Reuter, Renormalization group improved black hole space-times, Phys. Rev. D 62, 043008 (2000).

[16] F. Saueressig, N. Alkofer, G. D’Odorico, and F. Vidotto, Black holes in asymptotically safe gravity, Proc. Sci. FFP14 (2016) 174.

[17] J. M. Pawlowski and D. Stock, Quantum-improved Schwarzschild-(A)dS and Kerr-(A)dS spacetimes, Phys. Rev. D 98, 106008 (2018).

[18] A. Platania, Dynamical renormalization of black-hole spacetimes, Eur. Phys. J. C 79, 470 (2019).

[19] J.F. Donoghue, Leading Quantum Correction to the Newtonian Potential, Phys. Rev. Lett. 72, 2996 (1994).

[20] J. F. Donoghue, M. M. Ivanov, and A. Shkerin, EPFL lectures on general relativity as a quantum field theory, arXiv:1702.00319.

[21] A. Akhundov and A. Shiekh, A review of leading quantum gravitational corrections to Newtonian gravity, Electron. J. Theor. Phys. 5, 1 (2008).

[22] L. Bosma, B. Knorr, and F. Saueressig (to be published). 
[23] C. Wetterich, Exact evolution equation for the effective potential, Phys. Lett. B 301, 90 (1993).

[24] T. R. Morris, The exact renormalization group and approximate solutions, Int. J. Mod. Phys. A 09, 2411 (1994).

[25] J. Berges, N. Tetradis, and C. Wetterich, Nonperturbative renormalization flow in quantum field theory and statistical physics, Phys. Rep. 363, 223 (2002).

[26] J. Berges and D. Mesterhazy, Introduction to the nonequilibrium functional renormalization group, Nucl. Phys. B, Proc. Suppl. 228, 37 (2012).

[27] H. Gies, Introduction to the functional RG and applications to gauge theories, Lect. Notes Phys. 852, 287 (2012).

[28] M. Reuter, Nonperturbative evolution equation for quantum gravity, Phys. Rev. D 57, 971 (1998).

[29] A. Codello, R. Percacci, and C. Rahmede, Ultraviolet properties of f(R)-gravity, Int. J. Mod. Phys. A 23, 143 (2008).

[30] D. Benedetti, P. F. Machado, and F. Saueressig, Asymptotic safety in higher-derivative gravity, Mod. Phys. Lett. A 24, 2233 (2009).

[31] D. Benedetti, P. F. Machado, and F. Saueressig, Taming perturbative divergences in asymptotically safe gravity, Nucl. Phys. B824, 168 (2010).

[32] E. Manrique, M. Reuter, and F. Saueressig, Bimetric renormalization group flows in quantum Einstein gravity, Ann. Phys. (Amsterdam) 326, 463 (2011).

[33] D. Becker and M. Reuter, En route to background independence: Broken split-symmetry, and how to restore it with bi-metric average actions, Ann. Phys. (Amsterdam) 350, 225 (2014).

[34] H. Gies, B. Knorr, and S. Lippoldt, Generalized parametrization dependence in quantum gravity, Phys. Rev. D 92, 084020 (2015).

[35] N. Ohta, R. Percacci, and G. Paolo Vacca, Renormalization group equation and scaling solutions for $f(R)$ gravity in exponential parametrization, Eur. Phys. J. C 76, 46 (2016).

[36] H. Gies, B. Knorr, S. Lippoldt, and F. Saueressig, Gravitational Two-Loop Counterterm is Asymptotically Safe, Phys. Rev. Lett. 116, 211302 (2016).

[37] J. A. Dietz, T. R. Morris, and Z.H. Slade, Fixed point structure of the conformal factor field in quantum gravity, Phys. Rev. D 94, 124014 (2016).

[38] K. Falls, D. F. Litim, K. Nikolakopoulos, and C. Rahmede, On de Sitter solutions in asymptotically safe $f(R)$ theories, Classical Quantum Gravity 35, 135006 (2018).

[39] T. Denz, J. M. Pawlowski, and M. Reichert, Towards apparent convergence in asymptotically safe quantum gravity, Eur. Phys. J. C 78, 336 (2018).

[40] B. Knorr and S. Lippoldt, Correlation functions on a curved background, Phys. Rev. D 96, 065020 (2017).

[41] B. Knorr, Infinite order quantum-gravitational correlations, Classical Quantum Gravity 35, 115005 (2018).

[42] S. Gonzalez-Martin, T. R. Morris, and Z. H. Slade, Asymptotic solutions in asymptotic safety, Phys. Rev. D 95, 106010 (2017).

[43] K. Falls, C. R. King, D. F. Litim, K. Nikolakopoulos, and C. Rahmede, Asymptotic safety of quantum gravity beyond Ricci scalars, Phys. Rev. D 97, 086006 (2018).

[44] N. Christiansen, K. Falls, J. M. Pawlowski, and M. Reichert, Curvature dependence of quantum gravity, Phys. Rev. D 97, 046007 (2018).
[45] N. Alkofer and F. Saueressig, Asymptotically safe $f(R)$ gravity coupled to matter I: The polynomial case, Ann. Phys. (Amsterdam) 396, 173 (2018).

[46] N. Alkofer, Asymptotically safe $f(R)$-gravity coupled to matter II: Global solutions, Phys. Lett. B 789, 480 (2019).

[47] G. Gubitosi, R. Ooijer, C. Ripken, and F. Saueressig, Consistent early and late time cosmology from the RG flow of gravity, J. Cosmol. Astropart. Phys. 12 (2018) 004.

[48] K. G. Falls, D. F. Litim, and J. Schröder, Aspects of asymptotic safety for quantum gravity, Phys. Rev. D 99, 126015 (2019).

[49] A. Eichhorn, S. Lippoldt, J. M. Pawlowski, M. Reichert, and M. Schiffer, How perturbative is quantum gravity, Phys. Lett. B 792, 310 (2019).

[50] A. Eichhorn, P. Labus, J. M. Pawlowski, and M. Reichert, Effective universality in quantum gravity, SciPost Phys. 5, 031 (2018).

[51] P. Donà, A. Eichhorn, and R. Percacci, Matter matters in asymptotically safe quantum gravity, Phys. Rev. D 89, 084035 (2014).

[52] J. Meibohm and J. M. Pawlowski, Chiral fermions in asymptotically safe quantum gravity, Eur. Phys. J. C 76, 285 (2016).

[53] A. Eichhorn, A. Held, and J. M. Pawlowski, Quantumgravity effects on a Higgs-Yukawa model, Phys. Rev. D 94, 104027 (2016).

[54] A. Eichhorn and S. Lippoldt, Quantum gravity and standardmodel-like fermions, Phys. Lett. B 767, 142 (2017).

[55] N. Christiansen, D. F. Litim, J. M. Pawlowski, and M. Reichert, Asymptotic safety of gravity with matter, Phys. Rev. D 97, 106012 (2018).

[56] N. Christiansen and A. Eichhorn, An asymptotically safe solution to the U(1) triviality problem, Phys. Lett. B 770, 154 (2017).

[57] A. Eichhorn and A. Held, Top mass from asymptotic safety, Phys. Lett. B 777, 217 (2018).

[58] A. Eichhorn and F. Versteegen, Upper bound on the Abelian gauge coupling from asymptotic safety, J. High Energy Phys. 01 (2018) 030.

[59] A. Eichhorn, A. Held, and C. Wetterich, Quantum-gravity predictions for the fine-structure constant, Phys. Lett. B 782, 198 (2018).

[60] A. Eichhorn, S. Lippoldt, and M. Schiffer, Zooming in on fermions and quantum gravity, Phys. Rev. D 99, 086002 (2019).

[61] J. M. Pawlowski, M. Reichert, C. Wetterich, and M. Yamada, Higgs scalar potential in asymptotically safe quantum gravity, Phys. Rev. D 99, 086010 (2019).

[62] A. Eichhorn and A. Held, Mass Difference for Charged Quarks from Asymptotically Safe Quantum Gravity, Phys. Rev. Lett. 121, 151302 (2018).

[63] N. Christiansen, B. Knorr, J. M. Pawlowski, and A. Rodigast, Global flows in quantum gravity, Phys. Rev. D 93, 044036 (2016).

[64] N. Christiansen, B. Knorr, J. Meibohm, J. M. Pawlowski, and M. Reichert, Local quantum gravity, Phys. Rev. D 92, 121501(R) (2015).

[65] S. Weinberg, Critical phenomena for field theorists, in Erice Subnuclear Physics (Springer, Boston, 1976), p. 1. 
[66] M. Reuter and H. Weyer, Background independence and asymptotic safety in conformally reduced gravity, Phys. Rev. D 79, 105005 (2009).

[67] M. Reuter and H. Weyer, Conformal sector of quantum Einstein gravity in the local potential approximation: NonGaussian fixed point and a phase of unbroken diffeomorphism invariance, Phys. Rev. D 80, 025001 (2009).

[68] M. Demmel, F. Saueressig, and O. Zanusso, Fixedfunctionals of three-dimensional quantum Einstein gravity, J. High Energy Phys. 11 (2012) 131.

[69] M. Demmel, F. Saueressig, and O. Zanusso, RG flows of quantum Einstein gravity on maximally symmetric spaces, J. High Energy Phys. 06 (2014) 026.

[70] M. Demmel, F. Saueressig, and O. Zanusso, A proper fixed functional for four-dimensional Quantum Einstein Gravity, J. High Energy Phys. 08 (2015) 113.

[71] J. A. Dietz and T. R. Morris, Background independent exact renormalization group for conformally reduced gravity, J. High Energy Phys. 04 (2015) 118.

[72] A. Codello, R. Percacci, and C. Rahmede, Investigating the ultraviolet properties of gravity with a Wilsonian renormalization group equation, Ann. Phys. (Amsterdam) 324, 414 (2009).

[73] A. Codello and O. Zanusso, On the non-local heat kernel expansion, J. Math. Phys. (N.Y.) 54, 013513 (2013).

[74] J. Meibohm, J. M. Pawlowski, and M. Reichert, Asymptotic safety of gravity-matter systems, Phys. Rev. D 93, 084035 (2016).

[75] B. Knorr, C. Ripken, and F. Saueressig, Form factors in asymptotic safety: Conceptual ideas and computational toolbox, arXiv:1907.02903.

[76] M. Reuter and F. Saueressig, Renormalization group flow of quantum gravity in the Einstein-Hilbert truncation, Phys. Rev. D 65, 065016 (2002).

[77] J. Borchardt and B. Knorr, Global solutions of functional fixed point equations via pseudospectral methods, Phys. Rev. D 91, 105011 (2015); Erratum, Phys. Rev. D 93, 089904(E) (2016).

[78] J. Borchardt and B. Knorr, Solving functional flow equations with pseudo-spectral methods, Phys. Rev. D 94, 025027 (2016).
[79] J. P. Boyd, Orthogonal rational functions on a semi-infinite interval, J. Comput. Phys. 70, 63 (1987).

[80] J. P. Boyd, Chebyshev and Fourier Spectral Methods, 2nd ed. (Dover, New York, 2000).

[81] M. Reuter and H. Weyer, Quantum gravity at astrophysical distances, J. Cosmol. Astropart. Phys. 12 (2004) 001.

[82] F. Saueressig, G. Gubitosi, and C. Ripken, Scales and hierarchies in asymptotically safe quantum gravity: A review, arXiv:1901.01731.

[83] B. L. Giacchini, On the cancellation of Newtonian singularities in higher-derivative gravity, Phys. Lett. B 766, 306 (2017).

[84] X. Calmet and B. K. El-Menoufi, Quantum corrections to Schwarzschild black hole, Eur. Phys. J. C 77, 243 (2017).

[85] J. Edholm, A. S. Koshelev, and A. Mazumdar, Behavior of the Newtonian potential for ghost-free gravity and singularity-free gravity, Phys. Rev. D 94, 104033 (2016).

[86] T. Biswas, A. Mazumdar, and W. Siegel, Bouncing universes in string-inspired gravity, J. Cosmol. Astropart. Phys. 03 (2006) 009.

[87] L. Modesto, Super-renormalizable quantum gravity, Phys. Rev. D 86, 044005 (2012).

[88] T. Biswas, E. Gerwick, T. Koivisto, and A. Mazumdar, Towards Singularity and Ghost Free Theories of Gravity, Phys. Rev. Lett. 108, 031101 (2012).

[89] L. Modesto and L. Rachwal, Nonlocal quantum gravity: A review, Int. J. Mod. Phys. D 26, 1730020 (2017).

[90] A. Connes, Gravity coupled with matter and foundation of noncommutative geometry, Commun. Math. Phys. 182, 155 (1996).

[91] K. van den Dungen and W. D. van Suijlekom, Particle physics from almost commutative spacetimes, Rev. Math. Phys. 24, 1230004 (2012).

[92] A. Connes and M. Marcolli, Noncommutative Geometry, Quantum Fields and Motives (American Mathematical Society, 2007).

[93] M. A. Kurkov, F. Lizzi, and D. Vassilevich, High energy bosons do not propagate, Phys. Lett. B 731, 311 (2014).

[94] N. Alkofer, F. Saueressig, and O. Zanusso, Spectral dimensions from the spectral action, Phys. Rev. D 91, 025025 (2015). 